\title{
A Banach Principle for Semifinite von Neumann Algebras
}

\author{
Vladimir CHILIN ${ }^{\dagger}$ and Semyon LITVINOV ${ }^{\ddagger}$ \\ $\dagger$ Department of Mathematics, National University of Uzbekistan, Tashkent 700095, Uzbekistan \\ E-mail: chilin@ucd.uz \\ $¥$ Department of Mathematics, Pennsylvania State University, \\ 76 University Drive, Hazleton, PA 18202, USA \\ E-mail:snl2@psu.edu
}

Received November 25, 2005, in final form February 10, 2006; Published online February 20, 2006 Original article is available at http://www.emis.de/journals/SIGMA/2006/Paper023/

\begin{abstract}
Utilizing the notion of uniform equicontinuity for sequences of functions with the values in the space of measurable operators, we present a non-commutative version of the Banach Principle for $L^{\infty}$.
\end{abstract}

Key words: von Neumann algebra; measure topology; almost uniform convergence; uniform equicontinuity; Banach Principle

2000 Mathematics Subject Classification: 46L51

\section{Introduction}

Let $(\Omega, \Sigma, \mu)$ be a probability space. Denote by $\mathcal{L}=\mathcal{L}(\Omega, \mu)$ the set of all (classes of) complexvalued measurable functions on $\Omega$. Let $\tau_{\mu}$ stand for the measure topology in $\mathcal{L}$. The classical Banach Principle may be stated as follows.

Classical Banach Principle. Let $(X,\|\cdot\|)$ be a Banach space, and let $a_{n}:(X,\|\cdot\|) \rightarrow\left(\mathcal{L}, \tau_{\mu}\right)$ be a sequence of continuous linear maps. Consider the following properties:

(I) the sequence $\left\{a_{n}(x)\right\}$ converges almost everywhere (a.e.) for every $x \in X$;

(II) $a^{\star}(x)(\omega)=\sup _{n}\left|a_{n}(x)(\omega)\right|<\infty$ a.e. for every $x \in X$;

(III) (II) holds, and the maximal operator $a^{\star}:(X,\|\cdot\|) \rightarrow\left(\mathcal{L}, \tau_{\mu}\right)$ is continuous at 0 ;

(IV) the set $\left\{x \in X:\left\{a_{n}(x)\right\}\right.$ converges a.e. $\}$ is closed in $X$.

Implications $(\mathrm{I}) \Rightarrow(\mathrm{II}) \Rightarrow(\mathrm{III}) \Rightarrow(\mathrm{IV})$ always hold. If, in addition, there is a set $D \subset X$, $\bar{D}=X$, such that the sequence $\left\{a_{n}(x)\right\}$ converges a.e. for every $x \in D$, then all four conditions (I)-(IV) are equivalent.

The Banach Principle is most often and successfully applied in the context $X=\left(L^{p},\|\cdot\|_{p}\right)$, $1 \leq p<\infty$. At the same moment, in the case $p=\infty$ the uniform topology in $L^{\infty}$ appears to be too strong for the "classical" Banach Principle to be effective in $L^{\infty}$. For example, continuous functions are not uniformly dense in $L^{\infty}$.

In [1], employing the fact that the unit ball $L_{1}^{\infty}=\left\{x \in L^{\infty}:\|x\|_{\infty} \leq 1\right\}$ is complete in $\tau_{\mu}$, the authors suggest to consider the measure topology in $L^{\infty}$ replacing $(X,\|\cdot\|)$ by $\left(L_{1}^{\infty}, \tau_{\mu}\right)$. Note that, since $L_{1}^{\infty}$ is not a linear space, geometrical complications occur, which in [1] are treated with the help of the following lemma.

Lemma 1. If $N(x, \delta)=\left\{y \in L_{1}^{\infty}:\|y-x\|_{1} \leq \delta\right\}, x \in L_{1}^{\infty}, \delta>0$, then $N(0, \delta) \subset N(x, \delta)-$ $N(x, \delta)$ for any $x \in L_{1}^{\infty}, \delta>0$. 
An application of the Baire category theorem yields the following replacement of (I) $\Rightarrow$ (II).

Theorem 1 ([1]). Let $a_{n}: L^{\infty} \rightarrow \mathcal{L}$ be a sequence of $\tau_{\mu}$-continuous linear maps such that the sequence $\left\{a_{n}(x)\right\}$ converges a.e. for all $x \in L^{\infty}$. Then the maximal operator $a^{\star}(x)(\omega)=$ $\sup _{n}\left|a_{n}(x)(\omega)\right|, x \in L^{\infty}$, is $\tau_{\mu}$-continuous at 0 on $L_{1}^{\infty}$.

At the same time, as it is known [1], even for a sequence $a_{n}: L^{\infty} \rightarrow L^{\infty}$ of contractions, in which case condition (II) is clearly satisfied, the maximal operator $a^{\star}: L_{1}^{\infty} \rightarrow L_{1}^{\infty}$ may be not $\tau_{\mu}$-continuous at 0 , i.e., (II) does not necessarily imply (III), whereas a replacement of the implication (III) $\Rightarrow$ (IV) does hold:

Theorem 2 ([1]). Assume that each $a_{n}: L^{\infty} \rightarrow \mathcal{L}$ is linear, condition (II) holds with $X=L^{\infty}$, and the maximal operator $a^{\star}: L^{\infty} \rightarrow \mathcal{L}$ is $\tau_{\mu}$-continuous at 0 on $L_{1}^{\infty}$. Then the set $\left\{x \in L_{1}^{\infty}\right.$ : $\left\{a_{n}(x)\right\}$ converges a.e. $\}$ is closed in $\left(L_{1}^{\infty}, \tau_{\mu}\right)$.

A non-commutative Banach Principle for measurable operators affiliated with a semifinite von Neumann algebra was established in [5]. Then it was refined and applied in [7, 4, 3]. In [3] the notion of uniform equicontinuity of a sequence of functions into $L(M, \tau)$ was introduced. The aim of this study is to present a non-commutative extension of the Banach Principle for $L^{\infty}$ that was suggested in [1]. We were unable to prove a verbatim operator version of Lemma 1. Instead, we deal with the mentioned geometrical obstacles via essentially non-commutative techniques, which helps us to get rid of some restrictions in [1]. First, proof of Lemma 1 essentially depends on the assumption that the functions in $\mathcal{L}$ be real-valued while the argument of the present article does not employ this condition. Also, our approach eliminates the assumption of the finiteness of measure.

\section{Preliminaries}

Let $M$ be a semifinite von Neumann algebra acting on a Hilbert space $H$, and let $B(H)$ denote the algebra of all bounded linear operators on $H$. A densely-defined closed operator $x$ in $H$ is said to be affiliated with $M$ if $y^{\prime} x \subset x y^{\prime}$ for every $y^{\prime} \in B(H)$ with $y^{\prime} z=z y^{\prime}, z \in M$. We denote by $P(M)$ the complete lattice of all projections in $M$. Let $\tau$ be a faithful normal semifinite trace on $M$. If $I$ is the identity of $M$, denote $e^{\perp}=I-e, e \in P(M)$. An operator $x$ affiliated with $M$ is said to be $\tau$-measurable if for each $\epsilon>0$ there exists a projection $e \in P(M)$ with $\tau\left(e^{\perp}\right) \leq \epsilon$ such that $e H$ lies in the domain of the operator $x$. Let $L=L(M, \tau)$ stand for the set of all $\tau$-measurable operators affiliated with $M$. Denote $\|\cdot\|$ the uniform norm in $B(H)$. If for any given $\epsilon>0$ and $\delta>0$ one sets

$$
V(\epsilon, \delta)=\left\{x \in L:\|x e\| \leq \delta \text { for some } e \in P(M) \text { with } \tau\left(e^{\perp}\right) \leq \epsilon\right\},
$$

then the topology $t_{\tau}$ in $L$ defined by the family $\{V(\epsilon, \delta): \epsilon>0, \delta>0\}$ of neighborhoods of zero is called a measure topology.

Theorem 3 ([9], see also [8]). $\left(L, t_{\tau}\right)$ is a complete metrizable topological $*$-algebra.

Proposition 1. For any $d>0$, the sets $M_{d}=\{x \in M:\|x\| \leq d\}$ and $M_{d}^{h}=\left\{x \in M_{d}: x^{*}=x\right\}$ are $t_{\tau}$-complete.

Proof. Because $\left(L, t_{\tau}\right)$ is a complete metric space, it is enough to show that $M_{d}$ and $M_{d}^{h}$ are (sequentially) closed in $\left(L, t_{\tau}\right)$. If $M_{d} \ni x_{n} \rightarrow_{\tau} x \in L$, then $0 \leq x_{n}^{*} x_{n} \leq d \cdot I$ and, due to Theorem 3, $x_{n} x_{n}^{*} \rightarrow t_{\tau} x^{*} x$. Since $\{x \in L: x \geq 0\}$ is $t_{\tau^{-}}$-complete, we have $0 \leq x^{*} x \leq d \cdot I$, which implies that $x \in M_{d}$. Therefore, $M_{d}$ is closed in $\left(L, t_{\tau}\right)$. Similarly, it can be checked that $M_{d}^{h}$ is closed in $\left(L, t_{\tau}\right)$. 
A sequence $\left\{y_{n}\right\} \subset L$ is said to converge almost uniformly (a.u.) to $y \in L$ if for any given $\epsilon>0$ there exists a projection $e \in P(M)$ with $\tau\left(e^{\perp}\right) \leq \epsilon$ satisfying $\left\|\left(y-y_{n}\right) e\right\| \rightarrow 0$.

Proposition 2. If $\left\{y_{n}\right\} \subset L$, then the conditions

(i) $\left\{y_{n}\right\}$ converges a.u. in $L$;

(ii) for every $\epsilon>0$ there exists $e \in P(M)$ with $\tau\left(e^{\perp}\right) \leq \epsilon$ such that $\left\|\left(y_{m}-y_{n}\right) e\right\| \rightarrow 0$ as $m, n \rightarrow \infty$

are equivalent.

Proof. Implication (i) $\Rightarrow$ (ii) is trivial. (ii) $\Rightarrow$ (i): Condition (ii) implies that the sequence $\left\{y_{n}\right\}$ is fundamental in measure. Therefore, by Theorem 3, one can find $y \in L$ such that $y_{n} \rightarrow y$ in $t_{\tau}$. Fix $\epsilon>0$, and let $p \in P(M)$ be such that $\tau\left(p^{\perp}\right) \leq \epsilon / 2$ and $\left\|\left(y_{m}-y_{n}\right) p\right\| \rightarrow 0$ as $m, n \rightarrow \infty$. Because the operators $y_{n}, n \geq 1$, are measurable, it is possible to construct such a projection $q \in P(M)$ with $\tau\left(q^{\perp}\right) \leq \epsilon / 2$ that $\left\{y_{n} q\right\} \subset M$. Defining $e=p \wedge q$, we obtain $\tau\left(e^{\perp}\right) \leq \epsilon$, $y_{n} e=y_{n} q e \in M$, and

$$
\left\|y_{m} e-y_{n} e\right\|=\left\|\left(y_{m}-y_{n}\right) p e\right\| \leq\left\|\left(y_{m}-y_{n}\right) p\right\| \longrightarrow 0,
$$

$m, n \rightarrow \infty$. Thus, there exists $y(e) \in M$ satisfying $\left\|y_{n} e-y(e)\right\| \rightarrow 0$. In particular, $y_{n} e \rightarrow y(e)$ in $t_{\tau}$. On the other hand, $y_{n} e \rightarrow y e$ in $t_{\tau}$, which implies that $y(e)=y e$. Hence, $\left\|\left(y_{n}-y\right) e\right\| 0$, i.e. $y_{n} \rightarrow y$ a.u.

The following is a non-commutative Riesz's theorem [9]; see also [5].

Theorem 4. If $\left\{y_{n}\right\} \subset L$ and $y=t_{\tau}-\lim _{n \rightarrow \infty} y_{n}$, then $y=a . u$. $-\lim _{k \rightarrow \infty} y_{n_{k}}$ for some subsequence $\left\{y_{n_{k}}\right\} \subset\left\{y_{n}\right\}$.

\section{Uniform equicontinuity for sequences of maps into $L(M, \tau)$}

Let $E$ be any set. If $a_{n}: E \rightarrow L, x \in E$, and $b \in M$ are such that $\left\{a_{n}(x) b\right\} \subset M$, then we denote

$$
S(x, b)=S\left(\left\{a_{n}\right\}, x, b\right)=\sup _{n}\left\|a_{n}(x) b\right\| .
$$

Definition below is in part due to the following fact.

Lemma 2. Let $(X,+)$ be a semigroup, $a_{n}: X \rightarrow L$ be a sequence of additive maps. Assume that $\bar{x} \in X$ is such that for every $\epsilon>0$ there exist a sequence $\left\{x_{k}\right\} \subset X$ and a projection $p \in P(M)$ with $\tau\left(p^{\perp}\right) \leq \epsilon$ such that

(i) $\left\{a_{n}\left(\bar{x}+x_{k}\right)\right\}$ converges a.u. as $n \rightarrow \infty$ for every $k$;

(ii) $S\left(x_{k}, p\right) \rightarrow 0, k \rightarrow \infty$.

Then the sequence $\left\{a_{n}(\bar{x})\right\}$ converges a.u. in $L$.

Proof. Fix $\epsilon>0$, and let $\left\{x_{k}\right\} \subset X$ and $p \in P(M), \tau\left(p^{\perp}\right) \leq \epsilon / 2$, be such that conditions (i) and (ii) hold. Pick $\delta>0$ and let $k_{0}=k_{0}(\delta)$ be such that $S\left(x_{k_{0}}, p\right) \leq \delta / 3$. By Proposition 2, there is a projection $q \in P(M)$ with $\tau\left(q^{\perp}\right) \leq \epsilon / 2$ and a positive integer $N$ for which the inequality

$$
\left\|\left(a_{m}\left(\bar{x}+x_{k_{0}}\right)-a_{n}\left(\bar{x}+x_{k_{0}}\right)\right) q\right\| \leq \frac{\delta}{3}
$$

holds whenever $m, n \geq N$. If one defines $e=p \wedge q$, then $\tau\left(e^{\perp}\right) \leq \epsilon$ and

$$
\begin{aligned}
& \left\|\left(a_{m}(\bar{x})-a_{n}(\bar{x})\right) e\right\| \leq\left\|\left(a_{m}\left(\bar{x}+x_{k_{0}}\right)-a_{n}\left(\bar{x}+x_{k_{0}}\right)\right) e\right\| \\
& \quad+\left\|a_{m}\left(x_{k_{0}}\right) e\right\|+\left\|a_{n}\left(x_{k_{0}}\right) e\right\| \leq \delta
\end{aligned}
$$

for all $m, n \geq N$. Therefore, by Proposition 2, the sequence $\left\{a_{n}(\bar{x})\right\}$ converges a.u. in $L$. 
Let $(X, t)$ be a topological space, and let $a_{n}: X \rightarrow L$ and $x_{0} \in X$ be such that $a_{n}\left(x_{0}\right)=0$, $n=1,2, \ldots$. Recall that the sequence $\left\{a_{n}\right\}$ is equicontinuous at $x_{0}$ if, given $\epsilon>0$ and $\delta>0$, there is a neighborhood $U$ of $x_{0}$ in $(X, t)$ such that $a_{n} U \subset V(\epsilon, \delta), n=1,2, \ldots$, i.e., for every $x \in U$ and every $n$ one can find a projection $e=e(x, n) \in P(M)$ with $\tau\left(e^{\perp}\right) \leq \epsilon$ satisfying $\left\|a_{n}(x) e\right\| \leq \delta$.

Definition. Let $(X, t), a_{n}: X \rightarrow L$, and $x_{0} \in X$ be as above. Let $x_{0} \in E \subset X$. The sequence $\left\{a_{n}\right\}$ will be called uniformly equicontinuous at $x_{0}$ on $E$ if, given $\epsilon>0, \delta>0$, there is a neighborhood $U$ of $x_{0}$ in $(X, t)$ such that for every $x \in E \cap U$ there exists a projection $e=e(x) \in P(M), \tau\left(e^{\perp}\right) \leq \epsilon$, satisfying $S(x, e) \leq \delta$.

As it can be easily checked, the uniform equicontinuity is a non-commutative generalization of the continuity of the maximal operator, a number of equivalent forms of which are presented in [1].

Let $\rho$ be an invariant metric in $L$ compatible with $t_{\tau}$ (see Theorem 3 ).

Lemma 3. Let $d>0$. If a sequence $a_{n}: M \rightarrow L$ of additive maps is uniformly equicontinuous at 0 on $M_{d}^{h}$, then it is also uniformly equicontinuous at 0 on $M_{d}$.

Proof. Fix $\epsilon>0, \delta>0$. Let $\gamma>0$ be such that, given $x \in M_{d}^{h}, \rho(0, x)<\gamma$, there is $e=e(x) \in$ $P(M)$ for which $\tau\left(e^{\perp}\right) \leq \epsilon / 2$ and $S(x, e) \leq \delta / 2$ hold. Pick $x \in M_{d}$ with $\rho(0, x)<\gamma$. We have $x=\operatorname{Re}(x)+i \operatorname{Im}(x)$, where $\operatorname{Re}(x)=\frac{x+x^{*}}{2}, \operatorname{Im}(x)=\frac{x-x^{*}}{2 i}$. Clearly, $\operatorname{Re}(x), \operatorname{Im}(x) \in M_{d}^{h}$ and $\rho(0, \operatorname{Re}(x))<\gamma, \rho(0, \operatorname{Im}(x))<\gamma$. Therefore, one can find such $p, q \in P(M)$ with $\tau\left(p^{\perp}\right) \leq \epsilon / 2$ and $\tau\left(q^{\perp}\right) \leq \epsilon / 2$ that $S(\operatorname{Re}(x), p) \leq \delta / 2$ and $S(\operatorname{Im}(x), q) \leq \delta / 2$. Defining $r=p \wedge q$, we get $\tau\left(r^{\perp}\right) \leq \epsilon$ and

$$
S(x, r) \leq S(\operatorname{Re}(x), r)+S(\operatorname{Im}(x), r) \leq S(\operatorname{Re}(x), p)+S(\operatorname{Im}(x), q) \leq \delta,
$$

implying that the sequence $\left\{a_{n}\right\}$ is uniformly equicontinuous at 0 on $M_{d}$.

Lemma 4. Let a sequence $a_{n}: M \rightarrow L$ of additive maps be uniformly equicontinuous at 0 on $M_{d}$ for some $0<d \in \mathbb{Q}$. Then $\left\{a_{n}\right\}$ is also uniformly equicontinuous at 0 on $M_{s}$ for every $0<s \in \mathbb{Q}$.

Proof. Pick $0<s \in \mathbb{Q}$, and let $r=d / s$. Given $\epsilon>0, \delta>0$, one can present such $\gamma>0$ that for every $x \in M_{d}$ with $\rho(0, x)<\gamma r$ there is a projection $e=e(x) \in P(M), \tau\left(e^{\perp}\right) \leq \epsilon$, satisfying $S(x, e) \leq \delta r$. Since $a_{n}$ is additive and $d, s \in \mathbb{Q}$, we have $a_{n}(r x)=r a_{n}(x)$. Also, $r x \in M_{d}$ and $\rho(0, r x)<\gamma r$ is equivalent to $x \in M_{s}$ and $\rho(0, x)<\gamma$. Thus, given $x \in M_{s}$ with $\rho(0, x)<\gamma$, we have

$$
\left\|a_{n}(x) e\right\|=\frac{1}{r} \cdot\left\|a_{n}(r x) e\right\| \leq \delta
$$

meaning that the sequence $\left\{a_{n}\right\}$ is uniformly equicontinuous at 0 on $M_{s}$.

\section{Main results}

Let $0 \in E \subset M$. For a sequence of functions $a_{n}:\left(M, t_{\tau}\right) \rightarrow L$, consider the following conditions

$(\mathrm{CNV}(E))$ almost uniform convergence of $\left\{a_{n}(x)\right\}$ for every $x \in E$;

$(\mathrm{CNT}(E))$ uniform equicontinuity at 0 on $E$;

$(\mathrm{CLS}(E))$ closedness in $\left(E, t_{\tau}\right)$ of the set $C(E)=\left\{x \in E:\left\{a_{n}(x)\right\}\right.$ converges a.u. $\}$. 
In this section we will study relationships among the conditions $\left(\mathrm{CNV}\left(M_{1}\right)\right),\left(\mathrm{CNT}\left(M_{1}\right)\right)$, and $\left(\operatorname{CLS}\left(M_{1}\right)\right)$.

Remarks. 1. Following the classical scheme (see Introduction), one more condition can be added to this list, namely, a non-commutative counterpart of the existence of the maximal operator, which can be stated as [5]:

$(\mathrm{BND}(E))$ given $x \in E$ and $\epsilon>0$, there is $e \in P(M), \tau\left(e^{\perp}\right) \leq \epsilon$, with $S(x, e)<\infty$.

This condition can be called a pointwise uniform boundedness of $\left\{a_{n}\right\}$ on $E$. It can be easily verified that $(\mathrm{CNV}(E))$ implies $(\operatorname{BND}(E))$. But, as it was mentioned in Introduction, even in the commutative setting, $\left(\mathrm{BND}\left(M_{1}\right)\right)$ does not guarantee $\left(\mathrm{CNT}\left(M_{1}\right)\right)$.

2. If $a_{n}$ is additive for every $n$, then $(\operatorname{CNV}(M))$ follows from $\left(\operatorname{CNV}\left(M_{1}\right)\right)$.

3. If $E$ is closed in $\left(M, t_{\tau}\right)$ (for instance, if $E=M_{d}$, or $E=M_{d}^{h}$; see Proposition 1), then $(\mathrm{CLS}(E))$ is equivalent to the closedness of $C(E)$ in $\left(L, t_{\tau}\right)$.

In order to show that $\left(\mathrm{CNV}\left(M_{1}\right)\right)$ entails $\left(\mathrm{CNT}\left(M_{1}\right)\right)$, we will provide some auxiliary facts.

Lemma 5. For any $0 \leq x \in L$ and $e \in P(M), x \leq 2\left(e x e+e^{\perp} x e^{\perp}\right)$.

Proof. If $a=e-e^{\perp}$, then $a^{*}=a$, which implies that

$$
0 \leq a x a=e x e-e x e^{\perp}-e^{\perp} x e+e^{\perp} x e^{\perp} .
$$

Therefore, $e x e^{\perp}+e^{\perp} x e \leq e x e+e^{\perp} x e^{\perp}$, and we obtain

$$
x=\left(e+e^{\perp}\right) x\left(e+e^{\perp}\right) \leq 2\left(e x e+e^{\perp} x e^{\perp}\right) .
$$

For $y \in M$, denote $l(y)$ the projection on $\overline{y H}$, and let $r(y)=I-n(y)$, where $n(y)$ denotes the projection on $\{\xi \in H: y \xi=0\}$. It is easily checked that $l\left(y^{*}\right)=r(y)$, so, if $y^{*}=y$, one can define $s(y)=l(y)=r(y)$. The projections $l(y), r(y)$, and $s(y)$ are called, respectively, a left support of $y$, a right support of $y$, and a support of $y=y^{*}$. It is well-known that $l(y)$ and $r(y)$ are equivalent projections, in which case one writes $l(y) \sim r(y)$. In particular, $\tau(l(y))=\tau(r(y))$, $y \in M$. If $y^{*}=y \in M, y_{+}=\int_{0}^{\infty} \lambda d E_{\lambda}$, and $y_{-}=-\int_{-\infty}^{0} \lambda d E_{\lambda}$, where $\left\{E_{\lambda}\right\}$ is the spectral family of $y$, then we have $y=y_{+}-y_{-}, y_{+}=s\left(y_{+}\right) y s\left(y_{+}\right)$, and $y_{-}=-s\left(y_{+}\right)^{\perp} y s\left(y_{+}\right)^{\perp}$. The next lemma is, in a sense, a non-commutative replacement of Lemma 0.1.

Lemma 6. Let $y^{*}=y \in M,-I \leq y \leq I$. Denote $e_{+}=s\left(y_{+}\right)$. If $x \in M$ is such that $0 \leq x \leq I$, then

$$
-I \leq y-e_{+} x e_{+} \leq I \quad \text { and } \quad-I \leq y+e_{+}^{\perp} x e_{+}^{\perp} \leq I .
$$

Proof. Because $e_{+} x e_{+} \geq 0$, we have $y-e_{+} x e_{+} \leq y \leq I$; analogously, $-I \leq y+e_{+}^{\perp} x e_{+}^{\perp}$. On the other hand, since we obviously have $e_{+} x e_{+} \leq e_{+}, e_{+}^{\perp} x e_{+}^{\perp} \leq e_{+}^{\perp}, e_{+} y e_{+} \leq e_{+}$, and $e_{+}^{\perp} y e_{+}^{\perp} \geq-e_{+}^{\perp}$, one can write

$$
y-e_{+} x e_{+}=y_{+}-y_{-}-e_{+} x e_{+}=y_{+}+e_{+}^{\perp} y e_{+}^{\perp}-e_{+} x e_{+} \geq y_{+}-e_{+}^{\perp}-e_{+}=y_{+}-I \geq-I
$$

and

$$
y+e_{+}^{\perp} x e_{+}^{\perp}=e_{+} y e_{+}-y_{-}+e_{+}^{\perp} x e_{+}^{\perp} \leq e_{+}-y_{-}+e_{+}^{\perp}=I-y_{-} \leq I,
$$

which finishes the proof.

Lemma 7. $a V(\epsilon, \delta) b \subset V(2 \epsilon, \delta)$ for all $\epsilon>0, \delta>0$, and $a, b \in M_{1}$. 
Proof. Let $x \in V(\epsilon, \delta)$. There exists $e \in P(M)$ such that $\tau\left(e^{\perp}\right) \leq \epsilon$ and $\|x e\| \leq \delta$. If we denote $q=n\left(e^{\perp} b\right)$, then

$$
b q=\left(e+e^{\perp}\right) b q=e b q+e^{\perp} b n\left(e^{\perp} b\right)=e b q .
$$

Besides, we have $q^{\perp}=r\left(e^{\perp} b\right) \sim l\left(e^{\perp} b\right) \leq e^{\perp}$, which implies that $\tau\left(q^{\perp}\right) \leq \epsilon$. Now, if one defines $p=e \wedge q$, then $\tau\left(p^{\perp}\right) \leq 2 \epsilon$ and

$$
\| \text { axbp }\|=\| \text { axbqp }\|=\| \text { axebqp }\|\leq\| \text { axeb }\|\leq\| a\|\cdot\| x e\|\cdot\| b \| \leq \delta .
$$

Therefore, $a x b \in V(2 \epsilon, \delta)$.

Lemma 8 ([5]). Let $f$ be the spectral projection of $b \in M, 0 \leq b \leq I$, corresponding to the interval $[1 / 2,1]$. Then

(i) $\tau\left(f^{\perp}\right) \leq 2 \cdot \tau(I-b)$;

(ii) $f=b c$ for some $c \in M$ with $0 \leq c \leq 2 \cdot I$.

We will also need the following fundamental result.

Theorem 5 ([6]). Let $a: M \rightarrow M$ be a positive linear map such that $a(I) \leq I$. Then $a(x)^{2} \leq a\left(x^{2}\right)$ for every $x^{*}=x \in M$.

The next theorem represents a non-commutative extension of Theorem 1.

Theorem 6. Let $a_{n}: M \rightarrow L$ be a $\left(C N V\left(M_{1}\right)\right)$ sequence of positive $t_{\tau}$-continuous linear maps such that $a_{n}(I) \leq I, n=1,2, \ldots$ Then the sequence $\left\{a_{n}\right\}$ is also $\left(C N T\left(M_{1}\right)\right)$.

Proof. Fix $\epsilon>0$ and $\delta>0$. For $N \in \mathbb{N}$ define

$$
F_{N}=\left\{x \in M_{1}^{h}: \sup _{n \geq N}\left\|\left(a_{N}(x)-a_{n}(x)\right) b\right\| \leq \delta \text { for some } b \in M, 0 \leq b \leq I, \tau(I-b) \leq \epsilon\right\} .
$$

Show that the set $F_{N}$ is closed in $\left(M_{1}^{h}, \rho\right)$. Let $\left\{y_{m}\right\} \subset F_{N}$ and $\rho\left(y_{m}, \bar{x}\right) \rightarrow 0$ for some $\bar{x} \in L$. It follows from Proposition' 1 that $\bar{x} \in M_{1}^{h}$. We have $a_{1}\left(y_{m}\right) \rightarrow a_{1}(\bar{x})$ in $t_{\tau}$, which, by Theorems 3 and 4 , implies that there is a subsequence $\left\{y_{m}^{(1)}\right\} \subset\left\{y_{m}\right\}$ such that $a_{1}\left(y_{m}^{(1)}\right)^{*} \rightarrow a_{1}(\bar{x})^{*}$ a.u. Similarly, there is a subsequence $\left\{y_{m}^{(2)}\right\} \subset\left\{y_{m}^{(1)}\right\}$ for which $a_{2}\left(y_{m}^{(2)}\right)^{*} \rightarrow a_{2}(\bar{x})^{*}$ a.u. Repeating this process and defining $x_{m}=y_{m}^{(m)} \in F_{N}, m=1,2, \ldots$, we obtain

$$
a_{n}\left(x_{m}\right)^{*} \longrightarrow a_{n}(\bar{x})^{*} \text { a.u., } \quad m \rightarrow \infty, \quad n=1,2, \ldots
$$

By definition of $F_{N}$, there exists a sequence $\left\{b_{m}\right\} \subset M, 0 \leq b_{m} \leq I, \tau\left(I-b_{m}\right) \leq \epsilon$, such that $\sup _{n \geq N}\left\|\left(a_{N}\left(x_{m}\right)-a_{n}\left(x_{m}\right)\right) b_{m}\right\| \leq \delta$ for every $m$. Because $M_{1}$ is weakly compact, there are a subnet $\left\{b_{\alpha}\right\} \subset\left\{b_{m}\right\}$ and $b \in M$ such that $b_{\alpha} \rightarrow b$ weakly, i.e. $\left(b_{\alpha} \xi, \xi\right) \rightarrow(b \xi, \xi)$ for all $\xi \in H$. Clearly $0 \leq b \leq I$. Besides, by the well-known inequality (see, for example [2]),

$$
\tau(I-b) \leq \liminf _{\alpha} \tau\left(I-b_{\alpha}\right) \leq \epsilon .
$$

We shall show that $\sup _{n \geq N}\left\|\left(a_{N}(\bar{x})-a_{n}(\bar{x})\right) b\right\| \leq \delta$. Fix $n \geq N$. Since $a_{k}\left(x_{m}\right)^{*} \rightarrow a_{k}(\bar{x})^{*}$ a.u., $k=n, N$, given $\sigma>0$, there exists a projection $e \in P(M)$ with $\tau\left(e^{\perp}\right) \leq \sigma$ satisfying

$$
\left\|e\left(a_{k}\left(x_{m}\right)-a_{k}(\bar{x})\right)\right\|=\left\|\left(a_{k}\left(x_{m}\right)^{*}-a_{k}(\bar{x})^{*}\right) e\right\| \longrightarrow 0, \quad m \rightarrow \infty, \quad k=n, N .
$$

Show first that $\left\|e\left(a_{N}(\bar{x})-a_{n}(\bar{x})\right) b\right\| \leq \delta$. For every $\xi, \eta \in H$ we have

$$
\left|\left(e\left(\left(a_{N}\left(x_{m}\right)-a_{n}\left(x_{m}\right)\right) b_{m}-\left(a_{N}(\bar{x})-a_{n}(\bar{x})\right) b\right) \xi, \eta\right)\right|
$$




$$
\begin{aligned}
\leq & \left|\left(e\left(a_{N}\left(x_{m}\right)-a_{n}\left(x_{m}\right)-a_{N}(\bar{x})+a_{n}(\bar{x})\right) b_{m} \xi, \eta\right)\right| \\
& +\left|\left(\left(b_{m}-b\right) \xi,\left(a_{N}(\bar{x})^{*}-a_{n}(\bar{x})^{*}\right) e \eta\right)\right| .
\end{aligned}
$$

Fix $\gamma>0$ and choose $m_{0}$ be such that

$$
\left\|e\left(a_{k}\left(x_{m}\right)-a_{k}(\bar{x})\right)\right\|<\gamma, \quad k=n, N
$$

whenever $m \geq m_{0}$. Since $b_{\alpha} \rightarrow b$ weakly, one can find such an index $\alpha(\gamma)$ that

$$
\left|\left(\left(b_{\alpha}-b\right) \xi,\left(a_{N}(\bar{x})^{*}-a_{n}(\bar{x})^{*}\right) e \eta\right)\right|<\gamma
$$

as soon as $\alpha \geq \alpha(\gamma)$. Because $\left\{b_{\alpha}\right\}$ is a subnet of $\left\{b_{m}\right\}$, there is such an index $\alpha\left(m_{0}\right)$ that $\left\{b_{\alpha}\right\}_{\alpha \geq \alpha\left(m_{0}\right)} \subset\left\{b_{m}\right\}_{m \geq m_{0}}$. In particular, if $\alpha_{0} \geq \max \left\{\alpha(\gamma), \alpha\left(m_{0}\right)\right\}$, then $b_{\alpha_{0}}=b_{m_{1}}$ for some $m_{1} \geq m_{0}$. It follows now from (1)-(3) that, for all $\xi, \eta \in H$ with $\|\xi\|=\|\eta\|=1$, we have

$$
\begin{aligned}
& \left|\left(e\left(a_{N}(\bar{x})-a_{n}(\bar{x})\right) b \xi, \eta\right)\right| \leq\left|\left(e\left(a_{N}\left(x_{m_{1}}\right)-a_{n}\left(x_{m_{1}}\right)\right) b_{m_{1}} \xi, \eta\right)\right| \\
& \quad+\left|\left(e\left(a_{N}\left(x_{m_{1}}\right)-a_{n}\left(x_{m_{1}}\right)-a_{N}(\bar{x})+a_{n}(\bar{x})\right) b_{m_{1}} \xi, \eta\right)\right| \\
& \quad+\left|\left(\left(b_{m_{1}}-b\right) \xi,\left(a_{N}(\bar{x})^{*}-a_{n}(\bar{x})^{*}\right) e \eta\right)\right| \\
& \quad \leq \delta+\left\|e\left(a_{N}\left(x_{m_{1}}\right)-a_{N}(\bar{x})\right)\right\|+\left\|e\left(a_{n}\left(x_{m_{1}}\right)-a_{n}(\bar{x})\right)\right\|+\gamma<\delta+3 \gamma .
\end{aligned}
$$

Due to the arbitrariness of $\gamma>0$, we get

$$
\left\|e\left(a_{N}(\bar{x})-a_{n}(\bar{x})\right) b\right\|=\sup _{\|\xi\|=\|\eta\|=1}\left|\left(e\left(a_{N}(\bar{x})-a_{n}(\bar{x})\right) b \xi, \eta\right)\right| \leq \delta .
$$

Next, we choose $e_{j} \in P(M)$ such that $\tau\left(e_{j}^{\perp}\right) \leq \frac{1}{j}$ and

$$
\left\|e_{j}\left(a_{k}\left(x_{m}\right)-a_{k}(\bar{x})\right)\right\| \longrightarrow 0 \text { as } m \rightarrow \infty, \quad k=n, N ; \quad j=1,2, \ldots
$$

Since $e_{j} \rightarrow I$ weakly, $e_{j}\left(a_{N}(\bar{x})-a_{n}(\bar{x})\right) b \rightarrow\left(a_{N}(\bar{x})-a_{n}(\bar{x})\right) b$ weakly, therefore,

$$
\left\|\left(a_{N}(\bar{x})-a_{n}(\bar{x})\right) b\right\| \leq \limsup _{j \rightarrow \infty}\left\|e_{j}\left(a_{N}(\bar{x})-a_{n}(\bar{x})\right) b\right\| \leq \delta .
$$

Thus, for every $n \geq N$ the inequality $\left\|\left(a_{N}(\bar{x})-a_{n}(\bar{x})\right) b\right\| \leq \delta$ holds, which implies that $\bar{x} \in F_{N}$ and $\overline{F_{N}}=F_{N}$.

Further, as $\left\{a_{n}(x)\right\}$ converges a.u. for every $x \in M_{1}$, taking into account Proposition 2, we obtain

$$
M_{1}^{h}=\bigcup_{N=1}^{\infty} F_{N} .
$$

By Proposition 1, the metric space $\left(M_{1}^{h}, \rho\right)$ is complete. Therefore, using the Baire category theorem, one can present such $N_{0}$ that $F_{N_{0}}$ contains an open set. In other words, there exist $x_{0} \in F_{N_{0}}$ and $\gamma_{0} \geq 0$ such that for any $x \in M_{1}^{h}$ with $\rho\left(x_{0}, x\right)<\gamma_{0}$ it is possible to find $b_{x} \in M$, $0 \leq b_{x} \leq I$, satisfying $\tau\left(I-b_{x}\right) \leq \epsilon$ and

$$
\sup _{n \geq N_{0}}\left\|\left(a_{N_{0}}(x)-a_{n}(x)\right) b_{x}\right\| \leq \delta .
$$

Let $f_{x}$ be the spectral projection of $b_{x}$ corresponding to the interval $[1 / 2,1]$. Then, according to Lemma $8, \tau\left(f_{x}^{\perp}\right) \leq 2 \epsilon$ and

$$
\sup _{n \geq N_{0}}\left\|\left(a_{n_{0}}(x)-a_{n}(x)\right) f_{x}\right\| \leq 2 \delta
$$


whenever $x \in M_{1}^{h}$ and $\rho\left(x_{0}, x\right)<\gamma_{0}$. Since the multiplication in $L$ is continuous with respect to the measure topology, Lemma 7 allows us to choose $0<\gamma_{1}<\gamma_{0}$ in such a way that $\rho(0, x)<\gamma_{1}$ would imply $\rho\left(0, a x^{2} b\right)<\gamma_{0}$ for every $a, b \in M_{1}$. Denote $e_{+}=s\left(x_{0}^{+}\right)$. Because $a_{i}:(M, \rho) \rightarrow$ $\left(L, t_{\tau}\right)$ is continuous for each $i$, there exists such $0<\gamma_{2}<\gamma_{1}$ that, given $x \in M$ with $\rho(0, x)<\gamma_{2}$, it is possible to find such a projection $p \in P(M), \tau\left(p^{\perp}\right) \leq \epsilon$, that

$$
\left\|a_{i}\left(e_{+} x^{2} e_{+}\right) p\right\| \leq \delta \quad \text { and } \quad\left\|a_{i}\left(e_{+}^{\perp} x^{2} e_{+}^{\perp}\right) p\right\| \leq \delta,
$$

$i=1, \ldots, N_{0}$. Let $x \in M_{1}^{h}$ be such that $\rho(0, x)<\gamma_{2}$. Since $0 \leq x^{2} \leq I$, Lemma 6 yields

$$
-I \leq x_{0}-e_{+} x^{2} e_{+} \leq I \quad \text { and } \quad-I \leq x_{0}+e_{+}^{\perp} x^{2} e_{+}^{\perp} \leq I,
$$

so, we have

$$
y=x_{0}-e_{+} x^{2} e_{+} \in M_{1}^{h} \quad \text { and } \quad z=x_{0}+e_{+}^{\perp} x^{2} e_{+}^{\perp} \in M_{1}^{h} .
$$

Besides, $\rho\left(x_{0}, y\right)=\rho\left(0,-e_{+} x^{2} e_{+}\right)<\gamma_{0}$, which implies that there is $f_{1} \in P(M)$ such that $\tau\left(f_{1}^{\perp}\right) \leq 2 \epsilon$ and

$$
\sup _{n \geq N_{0}}\left\|\left(a_{N_{0}}(y)-a_{n}(y)\right) f_{1}\right\| \leq 2 \delta .
$$

Analogously, one finds $f_{2} \in P(M), \tau\left(f_{2}^{\perp}\right) \leq 2 \epsilon$, satisfying

$$
\sup _{n \geq N_{0}}\left\|\left(a_{N_{0}}(z)-a_{n}(z)\right) f_{2}\right\| \leq 2 \delta
$$

As $\rho(0, x)<\gamma_{2}$, there is $p \in P(M)$ with $\tau\left(p^{\perp}\right) \leq \epsilon$ such that the inequalities

$$
\left\|a_{i}\left(e_{+} x^{2} e_{+}\right) p\right\| \leq \delta \quad \text { and } \quad\left\|a_{i}\left(e_{+}^{\perp} x^{2} e_{+}^{\perp}\right) p\right\| \leq \delta
$$

hold for all $i=1, \ldots, N_{0}$. Let $e=f_{x_{0}} \wedge f_{1} \wedge f_{2} \wedge p$. Then we have $\tau\left(e^{\perp}\right) \leq 7 \epsilon$ and, for $n>N_{0}$,

$$
\begin{aligned}
& \left\|a_{n}\left(e_{+} x^{2} e_{+}\right) e\right\| \leq \|\left(a_{N_{0}}\left(x_{0}-e_{+} x^{2} e_{+}\right)-a_{n}\left(x_{0}-e_{+} x^{2} e_{+}\right)\right. \\
& \left.\quad+a_{n}\left(x_{0}\right)-a_{N_{0}}\left(x_{0}\right)+a_{N_{0}}\left(e_{+} x^{2} e_{+}\right)\right) e\|\leq\|\left(a_{N_{0}}(y)-a_{n}(y)\right) f_{1} e \| \\
& \quad+\left\|\left(a_{N_{0}}\left(x_{0}\right)-a_{n}\left(x_{0}\right)\right) f_{x_{0}} e\right\|+\left\|a_{N_{0}}\left(e_{+} x^{2} e_{+}\right) p e\right\| \leq 5 \delta .
\end{aligned}
$$

At the same time, if $n \in\left\{1, \ldots, N_{0}\right\}$, then $\left\|a_{n}\left(e_{+} x^{2} e_{+}\right) e\right\|=\left\|a_{n}\left(e_{+} x^{2} e_{+}\right) p e\right\| \leq \delta$, so

$$
\left\|a_{n}\left(e_{+} x^{2} e_{+}\right) e\right\| \leq 5 \delta, \quad n=1,2, \ldots
$$

Analogously,

$$
\left\|a_{n}\left(e_{+}^{\perp} x^{2} e_{+}^{\perp}\right) e\right\| \leq 5 \delta, \quad n=1,2, \ldots
$$

Next, by Lemma 5 , we can write $0 \leq x^{2} \leq 2\left(e_{+} x^{2} e_{+}+e_{+}^{\perp} x^{2} e_{+}^{\perp}\right)$. Since $a_{n}$ is positive for every $n$, applying Theorem 5, we obtain

$$
0 \leq e a_{n}(x)^{2} e \leq e a_{n}\left(x^{2}\right) e \leq 2\left(e a_{n}\left(e_{+} x^{2} e_{+}\right) e+e a_{n}\left(e_{+}^{\perp} x^{2} e_{+}^{\perp}\right) e\right) .
$$

Therefore,

$$
\left\|a_{n}(x) e\right\|^{2}=\left\|e a_{n}(x)^{2} e\right\| \leq 20 \delta, \quad n=1,2, \ldots
$$

Summarizing, given $\epsilon>0, \delta>0$, it is possible to find such $\gamma>0$ that for every $x \in M_{1}^{h}$ with $\rho(0, x)<\gamma$ there is a projection $e=e(x) \in P(M)$ such that $\tau\left(\epsilon^{\perp}\right) \leq 7 \epsilon$ and

$$
S(x, e)=\sup _{n}\left\|a_{n}(x) e\right\| \leq \sqrt{20 \delta} .
$$

Thus, the sequence $\left\{a_{n}\right\}$ is $\left(\operatorname{CNT}\left(M_{1}^{h}\right)\right)$, hence, by Lemma $3,\left(\operatorname{CNT}\left(M_{1}\right)\right)$. 
Now we shall present a non-commutative extension of Theorem 2.

Theorem 7. $A\left(\operatorname{CNT}\left(M_{1}\right)\right)$ sequence $a_{n}: M \rightarrow L$ of additive maps is also $\left(\operatorname{CLS}\left(M_{1}\right)\right)$.

Proof. Let $\bar{x}$ belong to the $t_{\tau}$-closure of $C\left(M_{1}\right)$. By Proposition $1, \bar{x} \in M_{1}$. Fix $\epsilon>0$. Since, by Lemma 4 , the sequence $\left\{a_{n}\right\}$ is $\left(\operatorname{CNT}\left(M_{2}\right)\right)$, for every $k \in \mathbb{N}$, there is $\gamma_{k}>0$ such that, given $x \in M_{2}$ with $\rho(0, x)<\gamma_{k}$, one can find a projection $p_{k}=p_{k}(x) \in P(M), \tau\left(p_{k}^{\perp}\right) \leq \epsilon / 2^{k}$, satisfying $S\left(x, p_{k}\right) \leq 1 / k$. Let a sequence $\left\{y_{n}\right\} \subset C\left(M_{1}\right)$ be such that $\rho\left(\bar{x}, y_{k}\right)<\gamma_{k}$. If we set $x_{k}=y_{k}-\bar{x}$, then $x_{k} \in M_{2}, \rho\left(0, x_{k}\right)=\rho\left(\bar{x}, x_{k}+\bar{x}\right)=\rho\left(\bar{x}, y_{k}\right)<\gamma_{k}$, and $\bar{x}+x_{k}=y_{k} \in C\left(M_{1}\right)$, $k=1,2, \ldots$ If $e_{k}=p_{k}\left(x_{k}\right)$, then $\tau\left(e_{k}^{\perp}\right) \leq \epsilon / 2^{k}$ and also $S\left(x_{k}, e_{k}\right) \leq 1 / k$. Defining $e=\wedge_{k=1}^{\infty}$, we obtain $\tau\left(e^{\perp}\right) \leq \epsilon$ and $S\left(x_{k}, e\right) \leq 1 / k$. Therefore, by Lemma 2, the sequence $\left\{a_{n}(\bar{x})\right\}$ converges a.u., i.e. $\bar{x} \in C\left(M_{1}\right)$.

The following is an immediate consequence of the previous results of this section.

Theorem 8. Let $a_{n}: M \rightarrow L$ be a sequence of positive $t_{\tau}$-continuous linear maps such that $a_{n}(I) \leq I, n=1,2, \ldots$ If $\left\{a_{n}\right\}$ is $(\mathrm{CNV}(D))$ with $D$ being $t_{\tau}$-dense in $M_{1}$, then conditions $\left(\mathrm{CNV}\left(M_{1}\right)\right),\left(\mathrm{CNT}\left(M_{1}\right)\right)$, and $\left(\mathrm{CLS}\left(M_{1}\right)\right)$ are equivalent.

\section{Conclusion}

First we would like to stress that, due to Theorem 6, when establishing the almost uniform convergence of a sequence $\left\{a_{n}(x)\right\}$ for all $x \in L^{\infty}(M, \tau)=M$, the uniform equicontinuity at 0 on $M_{1}$ of the sequence $\left\{a_{n}\right\}$ is assumed. Also, as it is noticed in [1], the above formulation is important because, for example, if $\left\{a_{n}\right\}$ are bounded operators in a non-commutative $L^{p}$-space, $1 \leq p<\infty$, one may want to show that not only do these operators fail to converge a.u., but they fail so badly that $\left\{a_{n}\right\}$ may fail to converge a.u. on any class of operators which is $t_{\tau}$-dense in $M$.

\section{Acknowledgements}

S. Litvinov is partially supported by the 2004 PSU RD Grant.

[1] Bellow A., Jones R.L., A Banach principle for $L^{\infty}$, Adv. Math., 1996, V.36, 155-172.

[2] Bratelli O., Robinson D.N., Operator algebras and quantum statistical mechanics, Berlin, Springer, 1979.

[3] Chilin V., Litvinov S., Uniform equicontinuity for sequences of homomorphisms into the ring of measurable operators, Methods Funct. Anal. Topology, submitted.

[4] Chilin V., Litvinov S., Skalski A., A few remarks in non-commutative ergodic theory, J. Operator Theory, 2005, V.53, 301-320.

[5] Goldstein M., Litvinov S., Banach principle in the space of $\tau$-measurable operators, Studia Math., 2000, V.143, 33-41.

[6] Kadison R.V., A generalized Schwarz inequality and algebraic invariants for operator algebras, Ann. of Math., 1952, V.56, 494-503.

[7] Litvinov S., Mukhamedov F., On individual subsequential ergodic theorem in von Neumann algebras, Studia Math., 2001, V.145, 55-62.

[8] Nelson E., Notes on non-commutative integration, J. Funct. Anal., 1974, V.15, 103-116.

[9] Segal I., A non-commutative extension of abstract integration, Ann. of Math., 1953, V.57, 401-457. 